\title{
Curvature controlled wetting in two dimensions
}

\section{Gil, Tamir; Mikheev, Lev V.}

Published in:

Physical Review E. Statistical, Nonlinear, and Soft Matter Physics

Link to article, DOI:

10.1103/PhysRevE.52.772

Publication date:

1995

Document Version

Publisher's PDF, also known as Version of record

Link back to DTU Orbit

Citation (APA):

Gil, T., \& Mikheev, L. V. (1995). Curvature controlled wetting in two dimensions. Physical Review E. Statistical, Nonlinear, and Soft Matter Physics, 52(1), 772-780. https://doi.org/10.1103/PhysRevE.52.772

\section{General rights}

Copyright and moral rights for the publications made accessible in the public portal are retained by the authors and/or other copyright owners and it is a condition of accessing publications that users recognise and abide by the legal requirements associated with these rights.

- Users may download and print one copy of any publication from the public portal for the purpose of private study or research.

- You may not further distribute the material or use it for any profit-making activity or commercial gain

- You may freely distribute the URL identifying the publication in the public portal

If you believe that this document breaches copyright please contact us providing details, and we will remove access to the work immediately and investigate your claim. 


\title{
Curvature controlled wetting in two dimensions
}

\author{
Tamir Gil \\ Department of Physical Chemistry, The Technical University of Denmark, DK-2800 Lyngby, Denmark \\ Lev V. Mikheev \\ Nordita, Blegdamsvej 17, DK-2100, Copenhagen $\varnothing$, Denmark
}

(Received 15 November 1994)

\begin{abstract}
A complete wetting transition at vanishing curvature of the substrate in two-dimensional circular geometry is studied by the transfer matrix method. We find an exact formal mapping of the partition function of the problem onto that of a (1+1)-dimensional wetting problem in planar geometry. As the radius of the substrate $r_{0} \rightarrow \infty$, the leading effect of the curvature is adding the Laplace pressure $\Pi_{L} \propto r_{0}^{-1}$ to the pressure balance in the film. At temperatures and pressures under which the wetting is complete in planar geometry, Laplace pressure suppresses divergence of the mean thickness of the wetting layer $l_{W}$, leading to a power law $l_{W} \propto r_{0}^{1 / 3}$. At a critical wetting transition of a planar substrate, curvature adds a relevant field; the corresponding multiscaling forms are readily available. The method allows for the systematic evaluation of corrections to the leading behavior; the next to the leading term reduces the thickness by an amount proportional to $r_{0}^{-1 / 3}$.
\end{abstract}

PACS number(s): 68.45.Gd, 87.22.Bt, 05.40.+j

\section{INTRODUCTION}

When two thermodynamic phases $\alpha$ and $\beta$ are close to coexistence, i.e., to a first-order phase transition line, the presence of a substrate strongly preferring one of the phases leads to singular wetting effects. The preferred phase $\beta$ tends to form a layer intruding between the substrate and the other phase $\alpha$, even when the latter is stable in the bulk. In the complete wetting regime the thickness $l_{W}$ of the layer diverges continuously, $l_{W} \rightarrow \infty$, as the bulk $\alpha \rightarrow \beta$ phase transition line is approached. This continuous line of surface critical points may terminate at a wetting transition point, which can be first order or critical (Fig. 1). Scaling description is achieved in terms of two orthogonal fields: one, pressurelike, $\Pi_{b}$, measures the difference in the grand canonical potentials per unit volume of the two bulk phases; the other, temperaturelike, $t$, is a generalized coordinate measuring the distance from the wetting transition point $\left(P_{W}, T_{W}\right)$ along the coexistence line in Fig. 1. Then a power law $l_{W} \propto \Pi_{b}^{-\Psi^{c}}, \Pi_{b}>0$, characterizes growth of the film in the complete wetting regime, while a critical wetting point can be described by a two-parameter scaling form

$$
l_{W} \sim t^{-\Psi} \Phi\left[\Pi_{b} / t^{\Delta}\right], \Phi(x)= \begin{cases}\text { const }, & x \rightarrow 0 \\ x^{-\Psi^{c}}, & x \rightarrow+\infty\end{cases}
$$

where $\Delta \equiv \Psi / \Psi^{c}$.

Wetting of a planar substrate has been extensively studied $[1,2]$. Large-scale critical fluctuations are generally believed to be those of the $d^{\prime}=(d-1)$-dimensional interface separating the wetting film from the bulk $\alpha$ phase. If $\sigma$ is the stiffness of the interface, then these fluctuations are usually described by the phenomenological capillary Hamiltonian

$$
\mathcal{H}_{\mathrm{int}}=\int d^{d^{\prime}} x\left\{\frac{1}{2} \sigma[\nabla l(\vec{x})]^{2}+\Pi_{b} l+V(l)\right\} .
$$

Here $l(\vec{x})$ is the local thickness of the wetting film at a given point $\vec{x}$ in the substrate. The first term in the Hamiltonian represents excess surface energy caused by an increase in the interfacial area due to inhomogeneities in the thickness and the second describes the excess bulk energy of the $\beta$ phase compared to that of the $\alpha$ phase.

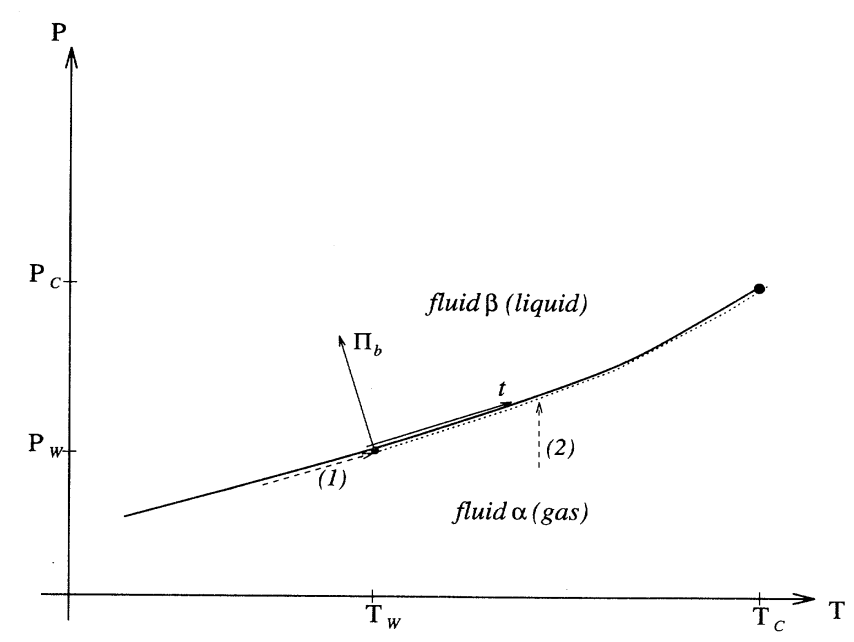

FIG. 1. Generic phase diagram for wetting by one of two fluid phases $(\beta)$ at the bulk coexistence line with the other $(\alpha)$ shown by thick solid line. The critical wetting transition occurs at $T_{W}$ by increasing the temperature along the gas-liquid coexistence curve, as shown by path (1). For $T>T_{W}$, any path taken in the direction of arrow (2) would terminate in a continuous complete wetting as the gas-liquid coexistence curve is approached. 
The last term describes effective interaction between the interface and the substrate, generally mediated by excitations in the $\beta$ phase [3]. It also contains a crucial hard core part, restricting $l$ to positive values.

In the planar geometry uniform changes in the thickness of the film do not change the area of the interface. Correspondingly, at coexistence of the two bulk phases $\Pi_{b}=0$, the equilibrium state of the film is determined by interplay of the interfacial potential $V$ and thermal fluctuations. In particular, nothing prevents the thickness from diverging when the effective interface-substrate interaction, resulting from renormalization of $V$ by thermal fluctuations, is repulsive. In many cases though, one may be interested in the role played by large but finite inclusions in a matrix undergoing a first-order transition (or phase separation). This question arises naturally in colloidal science, where colloidal particles may attract thick wetting layers of the preferred phase from a liquid mixture. Overlap between such wetting layers leads to an effective attraction between colloidal particles, which was conjectured to cause their aggregation [4].

In this paper we focus on the two-dimensional version of this problem: wetting around a disk embedded in a two-dimensional system undergoing a first-order phase transition (Fig. 2). One possible motivation comes from biophysics: integral protein macromolecules that are embedded in biomembranes [5,6]. Reconstructed membranes, composed of the main component of the biomembranes matrix (usually a certain type of a lipid molecule), often undergo first-order phase transitions (or phase separations) at temperatures that are similar to those in which the biomembranes can be found. One can thus envision that a large protein molecule can induce a thick wetting layer around it by selecting one of the matrix phases and that, just as in the case of colloids, the overlap between such layers may be an important factor favoring aggregation of proteins.

The crucial feature of the curved geometry comes from the fact that increasing the thickness of a wetting film does lead to an increase in the area or, in our twodimensional geometry, the length of the interface. The corresponding increase in the surface free energy suppresses complete wetting at $\Pi_{b}=0$. The situation can be qualitatively described using the picture of the equilibrium thickness of a wetting film being determined by the balance between the disjoining pressure [7] (to be precise one has to use a renormalized, i.e., averaged over thermal fluctuations, potential $V$ here)

$$
\Pi_{d}=\partial V / \partial l
$$

and the bulk compressing pressure $\Pi_{b}$. In the presence of curvature a term, the Laplace pressure

$$
\Pi_{L}=\sigma / r,
$$

where $r=r_{0}+l$ is the radius of curvature of the interface measured from the center of the substrate (Fig. 2), has to be added to the bulk part of the thinning pressure. For any bounded potential $V$ the disjoining pressure $\Pi_{d}$ falls off faster than $l^{-1}$, so that even at $\Pi_{b}=0$ the film cannot become infinite. This comes on top of the fact

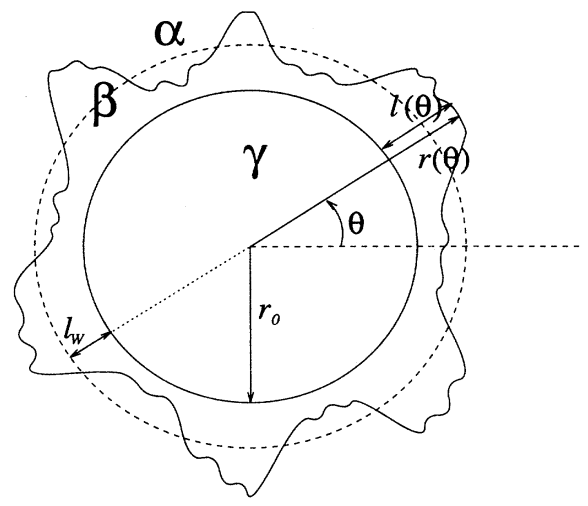

FIG. 2. The model under consideration: a circular solid substrate of radius $r_{0}$, a fluid phase $\alpha$, and a fluid phase $\beta$, away from the melting point of the substrate $(\gamma)$. At an angle $\theta$, measured from some arbitrary origin, $r(\theta)$ denotes the location of the interface measured from the center of the circle and $l(\theta)$ the same location measured from its surface. The mean thickness (dashed circle) is denoted by $l_{W}$.

that for any finite $r_{0}$ the system is finite, therefore the possibility of a genuine surface phase transition is very unlikely.

It is, however, possible to put the problem into the scaling framework by considering the limit of large radii $r_{0}$ of the substrate: if the planar substrate is completely wet, then the $r_{0} \rightarrow \infty$ limit is critical. From the formal point of view curvature is a relevant perturbation with respect to the critical or complete wetting fixed points of the renormalization group and we are interested in generalizing the scaling description (1) to the presence of this third relevant field. While in a real experiment curvature is normally fixed, as opposed to pressure and temperature, which are tunable, the fact that many interesting examples such as protein embeddings and colloidal particles involve values of $r_{0}$ much larger than any relevant length characterizing the bulk phases makes consideration of the singular limit $r_{0} \rightarrow \infty$ sensible. Note also that in the same limit $r_{0} \rightarrow \infty$ the length of the system is infinite, so that a surface phase transition is possible; in fact, we will see that the restrictions on divergence of the thickness imposed by the finite size are much weaker than those coming from the presence of Laplace pressure.

Wetting of cylindrical substrates has been considered in Refs. [8-10] within the mean-field approximation. While the analogy with the planar case suggests that the mean-field approximation should work reasonably well in the case of wetting in three dimensions controlled by the long-range van der Waals forces [1], it is unlikely to be accurate for the two dimensional problem. Wetting in two dimensions is known to be dominated by long-wavelength fluctuations of the interface, which are completely neglected in the mean-field approach.

In this paper we address the problem of the wetting of a disk in the transfer matrix framework, which is well known to provide a relatively easy and elegant solution for the planar $(d=2)$-dimensional wetting problem. We show how, by a suitable change of variables, the curved 
problem is mapped onto an equivalent planar one with a different set of parameters (Sec. III) and then use the results available for the latter problem to analyze the $r_{0} \rightarrow \infty$ limit of the former. It turns out (Sec. IV) that the leading behavior is correctly given by the phenomenological pressure balance (as developed further in Sec. II) if the asymptotic form $\Pi_{d} \propto l^{-3}$, well known for the planar case [11], is used. Our formalism, however, allows us to go further and to calculate corrections to the leading scaling (Sec. V), which are found to follow nontrivial power laws. Those corrections can presumably be quite important in defining the scaling domain and analyzing experimental data taken from a sample of finite radius $r_{0}$. We finish with summarizing the results and discussing possible implications of our findings.

\section{PHENOMENOLOGY: QUASIPLANAR APPROXIMATION}

Before analyzing the partition function in its general form, we present qualitative arguments that later turn out to describe the $r_{0} \rightarrow \infty$ limit correctly. These arguments are widely used when estimating the effect of thermal fluctuations of an interface in the vicinity of a planar wall (see [11-13]). For a macroscopic wetting layer of finite mean thickness $l_{W}$, one can define a longitudinal correlation length $\xi_{\|}$to describe the correlations between fluctuations in the value of the thickness $l$ along the interface [13]. We consider the limit

$$
r_{0} \gg \xi_{\|} \gg l_{W} \gg \xi_{b},
$$

which turns out to be self-consistent. Here we introduced $\xi_{b}$, the bulk correlation length, which is the smallest length scale at which treating an interface as a geometrical line still makes sense. Locally the substrate-interface system is then close to being planar and the whole system can be viewed as a collection of $2 \pi r_{0} / \xi_{\|}$independent planar segments. To study complete wetting controlled by $r_{0} \rightarrow \infty$, we consider $T>T_{W}$ and $\Pi_{b}=0$ (see Sec. I). Then we build a phenomenological approximation for the grand canonical potential $\Omega(l)$ by adding the self-energy of the interface $2 \pi \sigma\left(r_{0}+l\right)$ to the loss of entropy involved in preventing the interface from crossing the wall. In planar geometry, the latter results in the effective interaction

$$
V(l)=\text { const } /\left(\beta^{2} \sigma l^{2}\right)
$$

per unit length of the substrate [11-13], where $\beta \equiv k_{B} T$, $k_{B}$ being the Boltzmann constant. Multiplying (6) by the length $2 \pi r_{0}$, we obtain

$$
\Omega(l) \approx 2 \pi r_{0} \frac{\text { const }}{\beta^{2} \sigma l^{2}}+2 \pi \sigma\left(r_{0}+l\right) .
$$

The mean thickness $l_{W}$ is the value of $l$ that minimizes $\Omega$,

$$
l_{W}=\left[\text { const } \times 2 r_{0}(\beta \sigma)^{-2}\right]^{1 / 3} \propto(\beta \sigma)^{-2 / 3} r_{0}^{1 / 3} .
$$

The corresponding value of $\xi_{\|}$is

$$
\xi_{\|} \propto \beta \sigma l_{W}^{2} \propto(\beta \sigma)^{-1 / 3} r_{0}^{2 / 3}
$$

consistent with (5). All finite size effects are expected to be of the order of $\exp \left(-2 \pi r_{0} / \xi_{\|}\right) \propto \exp \left(-\operatorname{const} r_{0}^{1 / 3}\right)$, where $2 \pi r_{0}$ is the length of the circumference of the substrate. Thus, in the $r_{0} \rightarrow \infty$ limit, finite size effects vanish much (exponentially) faster than those of the Laplace pressure.

The main result (8) can be easily obtained in the pressure balance picture described in the Introduction: differentiation of the asymptotic expression (6) yields disjoining pressure $\Pi_{d} \propto \beta^{-2} \sigma^{-1} l^{-3}$. Balancing the latter against the Laplace pressure $\Pi_{L}=\sigma /\left(r_{0}+l\right) \approx \sigma / r_{0}$, where (5) has been used, one immediately arrives at (8).

The main assumption involved in this estimate is that the expression (6), which encapsulates nonperturbative effects of strong interfacial fluctuations, can still be used in the curved geometry under condition (5). In the remainder of the paper we will prove the validity of this assumption and calculate the leading corrections to the results of this section.

\section{TRANSFER MATRIX APPROACH}

Directly generalizing the ideas underlying the derivation of the interface Hamiltonian (2), one can quite generally write, for the $(d=2)$-dimensional wetting geometry of Fig. 2,

$$
\mathcal{H}=\int_{0}^{2 \pi}\left\{d \theta \frac{1}{2} \Pi_{b}\left[r^{2}(\theta)-{r_{0}}^{2}\right]+d L(\theta) \sigma+d u(r, \theta)\right\}
$$

The integrand includes (a) terms proportional to the area of the wetting layer $\left(\Pi_{b} / 2\right)\left(r^{2}(\theta)-r_{0}^{2}\right)$; (b) terms proportional to the length of the interface $L$ with the coefficient equal to the asymptotic, thickness-independent value of the stiffness $\sigma$; and (c) the substrate-interface interaction potential $u(r)$, which absorbs all thicknessdependent corrections to the first two terms. Note that we have made the usual assumption of no overhangs [known as solid on solid (SOS)] leading to single valued $r(\theta)$; the justification comes from the roughness exponent $\zeta$, defining how the amplitude of the thickness fluctuations scales with the correlation length $\delta l \propto \xi_{\|}^{\zeta}$, known to be equal to $\frac{1}{2}<1$ in the planar geometry [14].

To get some feeling for the last term in (10), we follow $[8,15]$ and calculate the substrate-interface interaction potential $u$ for the simplest model of molecules inside the $\beta$ and $\alpha$ phases interacting with those of the substrate via additive van der Waals pair potentials decaying as $\phi(r) \sim 1 / r^{p}$ with $p=6,7$ for nonretarded and retarded interactions, respectively. It is easily seen that as the thickness of the film $l=r-r_{0}$ increases relative to the radius of the substrate $r_{0}$, the interaction crosses over from the quasiplanar limit

$$
d u=d \theta r(\theta) V(l(\theta)) \propto d \theta \frac{r_{0}}{l^{p-3}}
$$


valid under condition (5), to the other limit $l \gg r_{0}$ in which the substrate is effectively reduced to a point so that $d u \propto r_{0}^{2} d \theta / l^{p-2}$. On the basis of the estimates of Sec. II, we expect the first of these two limits to be of relevance to us here and will use below scaling form

$$
d u=d \theta r(\theta) \tilde{V}\left(l(\theta), r_{0}\right)
$$

where the function $\tilde{V}\left(l(\theta), r_{0}\right)=V(l)\left[1+O\left(l / r_{0}\right)\right]$ contains the leading planar part plus power law corrections to it.

For a given interface configuration $r(\theta)$, the element of interfacial length

$$
d L(\theta)=\sqrt{r^{2}(\theta) d \theta^{2}+d r^{2}(\theta)} .
$$

Consistent with the SOS assumption made above, we expect fluctuations in the slope of the interface to be small

$$
\left|\frac{d r / d \theta}{r(\theta)}\right| \ll 1
$$

in the scaling limit. This allows a Taylor expansion of the element of length (13) in (10),

$$
\sigma d L(\theta) \approx d \theta\left[\sigma r(\theta)+\frac{\sigma}{2 r(\theta)}\left(\frac{d r}{d \theta}\right)^{2}\right]
$$

Substituting Eq. (15) into Eq. (10) [and keeping (5) in mind] we obtain

$$
\begin{aligned}
\mathcal{H}[r(\theta)]= & \int_{0}^{2 \pi} d \theta\left\{\frac{\sigma}{2 r(\theta)}\left(\frac{d r}{d \theta}\right)^{2}+\sigma r(\theta)\right. \\
& \left.+r(\theta) \tilde{V}\left[r(\theta)-r_{0}\right]+\frac{1}{2} \Pi_{b}\left(r^{2}(\theta)-r_{0}^{2}\right)\right\} \\
= & \int_{0}^{2 \pi} d \theta\left\{\frac{\sigma}{2 r(\theta)}\left(\frac{d r}{d \theta}\right)^{2}+v[r(\theta)]\right\},
\end{aligned}
$$

with $v[l(\theta)] \equiv \sigma r(\theta)+r \tilde{V}[r(\theta)]+\frac{1}{2} \Pi_{b}\left[r^{2}(\theta)-{r_{0}}^{2}\right]$ absorbing all terms without gradients. The partition function $\mathcal{Z}$ is given by

$$
\mathcal{Z}=\int \mathcal{D} r \exp \{-\beta \mathcal{H}[r(\theta)]\} .
$$

Here $\mathcal{D} r \equiv \mathcal{D}[r(\theta)]$ is the functional measure for the integration over all possible $r(\theta)$ functions satisfying the natural periodic boundary condition (Fig. 2)

$$
r(0)=r(2 \pi)
$$

and $\beta$ is the conventional notation for inverse temperature $1 /\left(k_{B} T\right)$.

The second line of Eq. (16) has the formal appearance of an interface Hamiltonian Eq. (2) describing a $(d=2)$ dimensional wetting of a finite planar substrate, except for the $r$ dependence of the coefficient at the gradient term. It is this possibility of a nonlinear feedback from the fluctuations of the interface into the stiffness term controlling those fluctuations that makes applicability of the relation (6) for the entropic repulsion doubtful in the curved geometry. Note that a much weaker dependence of stiffness on fluctuations in the thickness of a wetting layer has been recently shown to change critical wetting properties in three dimensions [16].

Nevertheless, we can formally eliminate the thickness dependence in the stiffness by simply transforming

$$
r(\theta) \rightarrow\left(\frac{\rho(\theta)}{2}\right)^{2},
$$

so that the term $\sigma(d r / d \theta)^{2} / 2 r(\theta)$ becomes $\sigma(d \rho / d \theta)^{2} / 2$. The Jacobian of the transformation $d r_{j} \rightarrow \rho_{j} d \rho_{j} / 2$ adds the term $-\ln (\rho / 2) / \beta$ to the integrand of Eq. (16) and Eq. (17) takes the form

$$
\begin{aligned}
\mathcal{Z}= & \int \mathcal{D} \rho \exp \left\{-\beta \int_{0}^{2 \pi} d \theta\left[\frac{\sigma}{2}\left(\frac{d \rho}{d \theta}\right)^{2}+v\left[\rho^{2}(\theta) / 4\right]\right.\right. \\
& \left.\left.-\frac{1}{\beta} \ln \left(\frac{\rho}{2}\right)\right]\right\} \\
& \equiv \int \mathcal{D} \rho e^{-\beta \mathcal{H}[\rho(\theta)]}
\end{aligned}
$$

where the last line defines a transformed Hamiltonian $\mathcal{H}[\rho(\theta)]$ governing fluctuations of the variable $\rho$.

This last expression is formally equivalent to a planar partition function and following Feynman's formulation [17], it can now be expressed as

$$
\mathcal{Z}=\int_{0}^{\infty} d \rho \mathcal{G}(\rho, 2 \pi ; \rho, 0)=\sum_{n} e^{-2 \pi \beta \varepsilon_{n}}
$$

Here

$$
\mathcal{G}(\rho, 2 \pi ; \rho, 0)=\sum_{n}\left|\phi_{n}(\rho)\right|^{2} e^{-2 \pi \beta \varepsilon_{n}}
$$

is the Green's function satisfying

$$
-\frac{1}{\beta} \frac{\partial \mathcal{G}\left(\rho, \theta ; \rho_{0}, 0\right)}{\partial \theta}=\hat{\mathcal{H}} \mathcal{G}\left(\rho, \rho_{0}\right) ;
$$

$\hat{\mathcal{H}}$ is the $\theta$-independent transfer matrix operator

$$
\hat{\mathcal{H}}=\frac{-1}{2 \beta^{2} \sigma} \partial_{\rho}^{2}+v(\rho)-\frac{1}{\beta} \ln \rho / 2
$$

its eigenfunctions $\phi_{n}(\rho)$ are solutions to the Schrödingerlike equation

$$
\hat{\mathcal{H}} \phi_{n}(\rho)=\varepsilon_{n} \phi_{n}(\rho),
$$

while the $\theta$ dependence of the Green's function comes through the exponential factors $\Theta_{n}=e^{-\beta \varepsilon_{n} \theta}$ satisfying $\rho$-independent differential equations

$$
-\frac{1}{\beta} \frac{\partial \Theta_{n}}{\partial \theta}=\varepsilon_{n} \Theta_{n}
$$

The boundary conditions, expressed in Eq. (18), are taken into consideration by setting $\rho=\rho(\theta=0)=\rho(\theta=$ $2 \pi$ ) in the argument of $\mathcal{G}$ in Eq. (21).

As a consistency check we apply this formulation to describe a circular domain of the $\beta$ phase inside an $\alpha$-phase matrix. Such domains can be viewed as an o rsimpli- 
fied description of the bulk fluctuations in the $\alpha$ phase at coexistence $\Pi_{b}=0$, so that the average radius $\bar{r}$ of such droplets has to give a rough estimate for the bulk correlation length in the system. Here we assume that we are not too far from the $\alpha-\beta$ critical bulk critical point $T_{c}$, so that the bulk correlation lengths in both phases are larger than the molecular size and are roughly equal to each other. Setting $\tilde{V}$ and $\Pi_{b}$ to zero in Eq. (20), we arrive at

$$
\mathcal{H}=\int_{0}^{\infty} d \theta\left\{\frac{\sigma}{2}\left(\frac{d \rho}{d \theta}\right)^{2}+\sigma \frac{\rho^{2}}{4}-\frac{1}{\beta} \ln \left(\frac{\rho}{2}\right)\right\} .
$$

The mean-field approximation for this model amounts to a minimization of

$$
\sigma \frac{\rho^{2}}{4}-\frac{1}{\beta} \ln \left(\frac{\rho}{2}\right)
$$

giving $\bar{\rho}^{2} / 4=1 /(\sigma \beta)$. It is easily seen, moreover, that all lengths in this model scale trivially with $1 /(\beta \sigma)$, so that one can write

$$
\xi_{b} \approx \bar{r}=(\bar{\rho} / 2)^{2}=\operatorname{const} \frac{1}{\beta \sigma},
$$

where $\bar{\rho}$ is the value of $\rho$ averaged over Eq. (20). The result (28) is consistent with the bulk hyperscaling relation $\sigma \xi_{b}^{d-1}=$ const $/ \beta[18]$, so that our formulation passes this test. We also note that the estimates of Sec. II can be rewritten using (28) as

$$
l_{W} \sim \xi_{b}\left(r_{0} / \xi_{b}\right)^{1 / 3}, \quad \xi_{\|} \sim \xi_{b}\left(r_{0} / \xi_{b}\right)^{2 / 3}
$$

so that as soon as $r_{0} \gg \xi_{b}$ the other inequalities in (5) are automatically satisfied.

We finish this section by introducing variables natural for this problem: we will measure the radial coordinate not from the origin, but from the substrate surface, as in $l=r-r_{0}$ (Fig. 2) and scale all lengths with $\xi_{b} \approx 1 /(\beta \sigma)$. The dimensionless variables $y$ and $y_{0}$ are hence defined by

$$
\begin{aligned}
y_{0} & \equiv \rho_{0} / \sqrt{\xi_{b}}=2 \sqrt{r_{0} / \xi_{b}} \\
\left(y_{0} y+y^{2} / 2\right) \frac{\xi_{b}}{2} & =r(\theta)-r_{0}=l(\theta)
\end{aligned}
$$

where $\rho(\theta)=\left(y_{0}+y\right) \sqrt{\xi_{b}}$.

Substituting Eq. (30) into Eq. (20), we obtain the dimensionless Hamiltonian

$$
\begin{aligned}
\beta \mathcal{H}= & \int_{0}^{2 \pi} d \theta\left\{\frac{1}{2}\left(\frac{d y}{d \theta}\right)^{2}+\frac{1}{4} y^{2}+\frac{1}{2} y_{0} y\right. \\
& +\frac{\Pi_{b}}{32 \sigma^{2} \beta}\left[y^{4}+4\left(y y_{0}^{3}+y^{3} y_{0}\right)+6 y^{2} y_{0}^{2}\right]+\beta u(y) \\
& \left.-\ln \left(1+\frac{y}{y_{0}}\right)\right\}+2 \pi\left[\frac{1}{4} y_{0}^{2}-\ln \left(\frac{y_{0}}{2}\right)\right]
\end{aligned}
$$

where the term in large square brackets is $y$ independent. The interface Hamiltonian approach is applicable only if the typical configurations of the interface satisfy

$$
l \gg \xi_{b}
$$

so that the intrinsic width of the interface can be neglected [14]. In our variables this implies $y \gg 1 / y_{0}$. Retaining only the leading terms we obtain the Hamilton operator (24)

$$
\begin{aligned}
\beta \hat{\mathcal{H}}= & -\frac{d^{2}}{d y^{2}}+\left(y_{0}+\frac{\Pi_{b}}{4 \sigma^{2} \beta} y_{0}^{3}\right) y+\left(\frac{1}{2}+\frac{3 \Pi_{b}}{8 \sigma^{2} \beta} y_{0}^{2}\right) y^{2} \\
& +\frac{\Pi_{b}}{4 \sigma^{2} \beta} y_{0} y^{3}+\beta u\left[y_{0} y+y^{2} / 2\right]
\end{aligned}
$$

\section{WETTING TRANSITIONS AS $\boldsymbol{R}_{0} \rightarrow \infty$}

\section{A. Planar limit: A recollection}

Having reduced the problem to the same form as the planar wetting problem, we are in a position now to apply the results available for the latter. For completeness we start with a brief recollection of these results (see Refs. $[19,20])$.

The functional integration in the partition function $\mathcal{Z}=\int \mathcal{D} l \exp \left\{-\beta \mathcal{H}_{\text {int }}[l]\right\}$ for the interface Hamiltonian (2) with periodic boundary conditions can be replaced by the eigenvalue problem for the operator

$$
\left[-\frac{1}{2 \beta^{2} \sigma} \frac{d^{2}}{d l^{2}}+\Pi_{b} l+V(l)\right] \psi(l)=\tilde{\varepsilon}_{n} \psi(l) .
$$

The partition function becomes

$$
\mathcal{Z}=\sum_{n} e^{-\beta L \tilde{\varepsilon}_{n}}
$$

where $L$ is the length of surface of the substrate, and the mean thickness is obtained via [20]

$$
l_{W}=-\frac{1}{\beta L} \sum_{n} \frac{\partial \tilde{\varepsilon}_{n}}{\partial \Pi_{b}} \frac{e^{-\beta L \tilde{\varepsilon}_{n}}}{\mathcal{Z}} .
$$

The thermodynamic limit is reached by taking $L$ to infinity; the free energy then becomes equal to the lowest "ground-state" eigenvalue of the transfer matrix $\tilde{\varepsilon}_{0}$ and $l_{W} \sim \partial \tilde{\varepsilon}_{0} / \partial \Pi_{b}$. It is therefore sufficient to investigate the ground state of the transfer matrix operator Eq. (34) in order to obtain the critical exponents of the wetting transitions; in the language of the quantum mechanical analogy, those transitions become transitions between having and not having a bound ground state.

It is convenient to factorize the interface potential $V=B U(l)$ in (34) into a dimensionless functional form $U(l)$ and an amplitude $B$ and then study various transitions as the amplitude $B$ varies with temperature. It has been shown [19] that attractive potentials $U(l) \leq 0$ with a finite first moment $\left|\int_{1}^{\infty} l U(l) d l\right|<\infty$ exhibit a transition from having to not having a bound ground state at a finite value of $B=B^{*}$. The singular part of the surface free energy vanishes as $\delta B^{2}$, while the meanthickness $l_{W}$ diverges $l_{W} \sim \delta B^{-\Psi}$ with $\Psi=1$ [cf. (1)], as $\delta B \equiv B-B^{*} \sim t \rightarrow 0+$. 
Power-law potentials $U(l)=-l^{-a}$ with $a>2$ have a finite second moment and thus behave as described above. The exponent $a=2$ is the marginal case still allowing for a wetting transition at finite $B$ [19]. In this case an infinite number of bound states become unbound at the transition point (for the quantum mechanical analogy, see Ref. [21]). For $a<2$ a wetting transition happens at $B \rightarrow 0$ and $\Psi=1 /|a-2|$. Finally, setting $a=-1$ and $B=\Pi_{b}$ one is able to model the complete wetting problem resulting in $\Psi^{c}=\Psi(a=-1)=1 / 3$.

These results can be combined into the two-parameter scaling form (1) [19] with

$$
\Phi(x)= \begin{cases}\text { const, } & x \rightarrow 0 \\ x^{-\Psi^{c}}, & x \rightarrow \infty\end{cases}
$$

with

$$
\begin{array}{ll}
\Psi=1, \Delta=3, & a>2 \\
\Psi=1 /|a-2|, \Delta=3 /|a-2|, & 0 \neq a<2 .
\end{array}
$$

\section{B. Mapping onto the planar scaling form}

In Secs. I and II we explained why wetting transitions can only occur in the limit of $r_{0} \rightarrow \infty$. According to Eq. (30) this would mean that $y_{0} \gg 1$. On the basis of the estimates of Sec. II it is reasonable to consider the limit $y_{0} \gg \bar{y}$, so that

$$
l_{W} \approx \frac{\xi_{b}}{2} y_{0} \bar{y}
$$

The transfer matrix operator of Eq. (33) in this limit is reduced to

$$
\beta \hat{\mathcal{H}}=-\frac{d^{2}}{d y^{2}}+\left(y_{0}+\frac{\Pi_{b}}{4 \sigma^{2} \beta} y_{0}^{3}\right) y+\beta u\left[y_{0} y\right]
$$

Dividing Eq. (40) by $y_{0}^{2}$ and transforming it back to the original radial coordinates in the limit of Eq. (39), i.e., $l=\xi_{b} y_{0} \bar{y} / 2$ and $r_{0}=\xi_{b} y_{0}^{2} / 4$, the Schrödinger equation (25) is transformed into

$$
\begin{aligned}
\frac{\hat{\mathcal{H}}}{r_{0}} \phi_{n}(l) & =\left[-\frac{1}{2 \beta^{2} \sigma} \frac{d^{2}}{d l^{2}}+\Pi_{\text {eff }} l+u(l) / r_{0}\right] \phi_{n}(l) \\
& =\left(\varepsilon_{n} / r_{0}\right) \phi_{n}(l),
\end{aligned}
$$

where

$$
\Pi_{\mathrm{eff}} \equiv \frac{\sigma}{r_{0}}+\Pi_{b}
$$

With $\mathcal{Z}$ given by Eq. (17), $\mathcal{H}$ being the Hamiltonian corresponding to $\hat{\mathcal{H}}$ in Eq. (41), the mean thickness $l_{W}$ is defined by

$$
l_{W}=-\frac{1}{\beta 2 \pi r_{0} \mathcal{Z}} \frac{\partial \mathcal{Z}}{\partial \Pi_{\mathrm{eff}}},
$$

while Eq. (21) yields the partition function

$$
\mathcal{Z}=\sum_{n} e^{-2 \pi r_{0} \beta \varepsilon_{n} / r_{0}} .
$$

The combination of the two gives rise to

$$
l_{W}=\frac{1}{\mathcal{Z}} \sum_{n} \frac{\partial\left(\varepsilon_{n} / r_{0}\right)}{\partial \Pi_{\mathrm{eff}}} e^{-2 \pi r_{0} \beta \varepsilon_{n} / r_{0}}
$$

We now observe that by transforming

$$
\begin{aligned}
2 \pi r_{0} & \leftrightarrow L, \\
\Pi_{\text {eff }} & \leftrightarrow \Pi_{b}, \\
\varepsilon_{n} / r_{0} & \leftrightarrow \tilde{\varepsilon}_{n}, \\
u(l) / r_{0} & \leftrightarrow V(l),
\end{aligned}
$$

we can map Eqs. (41)-(44) of the circular geometry onto Eqs. (34)-(36) of the planar geometry. We see that taking $r_{0}$ to infinity is equivalent to taking $L$ to infinity. Note, however, that since $\varepsilon_{n}$ in Eq. (45) is divided by $r_{0}$, the thermodynamic limit can be obtained from the groundstate eigenvalue only, if $\beta\left(\varepsilon_{1}-\varepsilon_{0}\right) \rightarrow \infty$ as $r_{0} \rightarrow \infty$.

To obtain the energy gap $\varepsilon_{1}-\varepsilon_{0}$ for when the ground state becomes unbound, we consider the simplest step potential

$$
u(l) / r_{0}= \begin{cases}+\infty, & l \leq 0 \\ 0, & l>0\end{cases}
$$

which is known to capture all essential features of the complete wetting phenomena in the $d=2$ planar geometry. Defining a dimensionless variable

$$
\zeta_{n} \equiv\left(2 \beta^{2} \sigma\right)^{1 / 3} \frac{\left(\varepsilon_{n} / r_{0}\right)-\Pi_{\mathrm{eff}} l}{\Pi_{\mathrm{eff}}^{2 / 3}}
$$

Eq. (41) becomes

$$
\left[\partial_{\zeta}^{2}+\zeta_{n}\right] \phi_{n}(\zeta)=0
$$

with the boundary condition

$$
\phi_{n}[\zeta(l=0)] \equiv 0,
$$

which is imposed by the potential (47). Equation (49) is solved by

$$
\phi_{n}(\zeta) \propto \operatorname{Ai}\left(-\zeta_{n}\right)
$$

where $\mathrm{Ai}$ is the Airy function.

For $\zeta>0$, corresponding to $l<\varepsilon /\left(r_{0} \Pi_{\mathrm{eff}}^{2 / 3}\right)$, the function $\operatorname{Ai}(-\zeta)$ vanishes at an infinite set of points $\left\{z_{n}\right\}$ satisfying

$$
J_{1 / 3}\left(z_{n}\right)=-J_{-1 / 3}\left(z_{n}\right)
$$

where $z \equiv \frac{2}{3} \zeta^{3 / 2}$. This set can be obtained numerically; it corresponds to an infinite set of energy eigenvalues $\left\{\varepsilon_{n}\right\}$ through the boundary conditions (50), i.e.,

$$
\varepsilon_{n} / r_{0}=\left(\frac{3}{2}\right)^{2 / 3} z_{n}^{2 / 3}\left(2 \beta^{2} \sigma\right)^{-1 / 3} \Pi_{\mathrm{eff}}^{2 / 3}
$$

The asymptotic expression for $\phi(\zeta)$ at large positive values of $\zeta$ is [21] 


$$
\phi(\zeta) \propto \zeta^{-1 / 4} \sin \left(\frac{2}{3} \zeta^{3 / 2}+\frac{\pi}{4}\right)
$$

In this limit, Eq. (50) can be satisfied by

$$
\varepsilon_{n} / r_{0}=\left(2 \beta^{2} \sigma\right)^{1 / 3}\left[\frac{3(2 n-1) \pi}{8}\right]^{2 / 3} \Pi_{\mathrm{eff}}^{2 / 3}
$$

implying that, at fixed temperature,

$$
\varepsilon_{n+1}-\varepsilon_{n} \sim r_{0} \Pi_{\mathrm{eff}}^{2 / 3} \sim r_{0}^{1 / 3}
$$

and, in particular, the gap

$$
\beta\left(\varepsilon_{1}-\varepsilon_{0}\right) \geq \beta\left(\varepsilon_{n+1}-\varepsilon_{n}\right) \sim r_{0}^{1 / 3} \stackrel{r_{0} \rightarrow \infty}{\gg} 1,
$$

which means that as $r_{0} \rightarrow \infty$ the ground state dominates the thermodynamical properties of the system.

The scaling form of Eqs. (37) and (38) with $\delta B<$ 0 can now be applied to the circular geometry by the transformations (46). In the leading order we obtain

$$
l_{W} \sim \Pi_{\mathrm{eff}}^{-1 / 3}=\left[\frac{\sigma}{r_{0}}\left(1+\frac{\Pi_{b} r_{0}}{\sigma}\right)\right]^{-1 / 3},
$$

which can be represented in the scaling form

$$
l_{W}=\sigma^{-1 / 3} r_{0}^{1 / 3} Y\left(\Pi_{b} r_{0} / \sigma\right), Y(x) \sim \begin{cases}\text { const, } & x=0 \\ x^{-1 / 3}, & x \rightarrow \infty\end{cases}
$$

At $\Pi_{b}=0$ we recover the result of Eq. (8). We proceed to use the relation between the gap in the transfer matrix spectrum and the correlation length [11]

$$
\xi_{\|}=r_{0}\left(\varepsilon_{1}-\varepsilon_{0}\right)^{-1} / \beta,
$$

which combined with (57) again yields the result (9) obtained earlier within the simple picture of Sec. II.

It is easily seen now that one can add an attractive part to the repulsive potential (47) and, using the asymptotic representation (11), show that the leading threeparameter scaling with $t, \Pi_{b} \rightarrow 0$, and $r_{0} \rightarrow \infty$ is obtained from the result for the planar geometry (1) by simply substituting $\Pi_{\text {eff }}$ as defined in (58) for $\Pi_{b}$.

\section{CORRECTIONS TO THE CRITICAL BEHAVIOR}

So far, the transfer matrix method only allowed us to confirm the results obtained from qualitative considerations earlier. The real power of the method, however, is in its ability to use the quantum mechanics perturbation theory to generate corrections to the leading scaling behavior. Such corrections can presumably be quite important in the analysis of a real experiment on a substrate of finite radius.

To generate the corrections we add next to leading terms in the limit $r_{0} \gg l_{W} \gg \xi_{b}$ and $y_{0} \gg \bar{y} \gg 1$ [see Eq. (39)] from Eq. (33) to the transfer matrix operator Eq. (40). This results in $\left[-\frac{d^{2}}{d y^{2}}+h_{1} y+h_{2} y^{2}+2 \beta u(y)\right] \phi_{n}(y)=E_{n} \phi_{n}(y)$

where $E_{n} \equiv 2 \beta \varepsilon_{n}, h_{1} \equiv y_{0}+\Pi_{b} y_{0}^{3} /\left(4 \sigma^{2} \beta\right), h_{2} \equiv[1+$ $\left.3 \Pi_{b} y_{0}^{2} /\left(4 \sigma^{2} \beta\right)\right] / 2$, and, in the limit of interest,

$$
h_{2} \bar{y}^{2} \ll h_{1} \bar{y} .
$$

The study of complete wetting is done as before with the hard wall potential (47) and the average value of $y$ is obtained from Eq. (45) by substituting $h_{1}$ for $\Pi_{b}$.

To first order in $y$, Eq. (61) is solved by $\phi_{n}(\zeta)$ in Eq. (51) but with $\zeta_{n}$ defined by

$$
\zeta_{n}=\frac{E_{n}-h_{1} y}{h_{1}^{2 / 3}} .
$$

The analogies to Eqs. (53)-(56) lead to

$$
z_{n}=\frac{2}{3}\left(\frac{E_{n}}{h_{1}^{2 / 3}}\right)^{3 / 2}
$$

and the asymptotic values

$$
\begin{gathered}
E_{n}=\left[\frac{3(2 n-1) \pi}{8}\right]^{2 / 3} h_{1}^{2 / 3}, \\
E_{1}-E_{0} \geq E_{n+1}-E_{n} \sim h_{1}^{2 / 3} \gg 1
\end{gathered}
$$

in the limit $y_{0} \rightarrow \infty$, so that

$$
\bar{y} \approx \frac{1}{\mathcal{Z}} \frac{\partial E_{0}}{\partial h_{1}} e^{-\pi E_{0}} \sim h_{1}^{-1 / 3}
$$

To obtain corrections to the above result, we apply the perturbation theory [21] and calculate a correction $\tilde{E}_{0}$ to the above $E_{0}$ due to the additional $h_{2} y^{2}$ term in Eq. (61). However, power-law tails in the asymptotic form (11) $u(l) \propto r_{0} / l^{p-3}(p=6,7$ for nonretarded and retarded interactions, respectively) of the interface potential (12) may invalidate the proposed complete wetting description. To control that, we add the relevant leading term in $u(y)$ by transforming

$$
\begin{gathered}
\beta u(l) \approx-\frac{r_{0} B}{l^{a}} \rightarrow-\frac{\xi_{b}^{1-a} B 2^{2(a-1)}}{y_{0}^{-2}\left(y^{2}+2 y y_{0}\right)^{a}} \\
\approx-\frac{\xi_{b}^{1-a} B 2^{a-2}}{y_{0}^{a-2} y^{a}} \approx \beta u(y) \\
\Rightarrow 2 \beta u(y) \approx-W / y^{-a} \\
W \equiv 2 \xi_{b}^{1-a} B\left(\frac{2}{y_{0}}\right)^{a-2}
\end{gathered}
$$

where $B$ is the factor discussed in Sec. IV A.

Now, to the lowest order, the corrections to the transfer matrix eigenvalues are given by [21]

$$
\tilde{E_{n}}=\int_{0}^{\infty} d y\left\{\phi_{n}^{*}(y)\left[h_{2} y^{2}+2 \beta u(y)\right] \phi_{n}(y)\right\}
$$


and in the limit of Eq. (62), we expect $\left|\tilde{E}_{n}\right| \ll\left|E_{n}\right|$, so that

$$
\left(E_{1}+\tilde{E}_{1}\right)-\left(E_{0}+\tilde{E}_{0}\right) \approx E_{1}-E_{0} \gg 1,
$$

and $\bar{y}$ can still be obtained by [see Eq. (67)]

$$
\bar{y} \approx \frac{e^{-\pi\left(E_{0}+\tilde{E}_{0}\right)}}{\mathcal{Z}}\left(\frac{\partial E_{0}}{\partial h_{1}}+\frac{\partial \tilde{E}_{0}}{\partial h_{1}}\right) .
$$

Upon using the definitions (63) and (51), Eqs. (68) and (69) imply

$$
\begin{aligned}
\tilde{E}_{0}= & -C^{2} \int_{\zeta_{0}(y=0)}^{\zeta_{0}(y=\infty)} h_{1}^{-1 / 3} d \zeta|\operatorname{Ai}(-\zeta)|^{2} \\
& \times\left[h_{2} h_{1}^{-2 / 3}\left(c_{0}-\zeta\right)^{2}-W h_{1}^{a / 3} \frac{1}{\left(c_{0}-\zeta\right)^{a}}\right],
\end{aligned}
$$

where $c_{0} \equiv \zeta_{0}(y=0)=E_{0} / h_{1}^{2 / 3}=\left(3 z_{0} / 2\right)^{2 / 3} \approx 2.339$ is a constant defined by Eqs. (52), (63), and (64). $C \equiv$ $\phi_{0}(\zeta) / \operatorname{Ai}\left(-\zeta_{0}\right)$ is a normalizing constant [see Eq. (51)] given by

$$
\begin{aligned}
1 & =\int_{0}^{\infty} d y\left|\phi_{0}\right|^{2} \\
& =-C^{2} h_{1}^{-1 / 3} \int_{\zeta_{0}(y=0)}^{\zeta_{0}(y=\infty)} d \zeta|\operatorname{Ai}(-\zeta)|^{2} \\
& \Rightarrow C^{2}=h_{1}^{1 / 3}\left[\int_{\zeta_{0}(y=\infty)}^{\zeta_{0}(y=0)} d \zeta|\operatorname{Ai}(-\zeta)|^{2}\right]^{-1} \sim h_{1}^{1 / 3} .
\end{aligned}
$$

The boundaries $\zeta_{0}(y=0)=c_{0}$ and $\zeta_{0}(y=\infty)=-\infty$ of the integrals (72) and (73) are universal and do not depend on the parameters of the problem. Those integrals converge and add numerical prefactors to the corresponding terms. Consequently, we obtain

$$
\tilde{E}_{0}=c_{1} h_{2} / h_{1}^{2 / 3}-c_{2} W h_{1}^{a / 3},
$$

where $c_{1}$ and $c_{2}$ are the nonscaling integrals

$$
\begin{aligned}
& c_{1} \equiv C^{2} h_{1}^{-1 / 3} \int_{-\infty}^{c_{0}} d \zeta\left[|\operatorname{Ai}(-\zeta)|^{2}\left(c_{0}-\zeta\right)^{2}\right] \\
& c_{2} \equiv C^{2} h_{1}^{-1 / 3} \int_{-\infty}^{c_{0}} d \zeta\left[|\operatorname{Ai}(-\zeta)|^{2} /\left(c_{0}-\zeta\right)^{a}\right]
\end{aligned}
$$

where $c_{1}>c_{2}>0$ for $a>-2$.

Equation (71) now yields

$$
\bar{y} \sim h_{1}^{-1 / 3}-\operatorname{const}\left(\frac{2}{3} c_{1} h_{2} h_{1}^{-5 / 3}+\frac{a}{3} c_{2} W h_{1}^{(a-3) / 3}\right),
$$

where the constant sets the ratio between the leading term and the corrections. Hence [see Eqs. (39), (58), and (68)]

$$
\begin{aligned}
l_{W} \sim & \left(\beta^{2} \sigma \Pi_{\mathrm{eff}}\right)^{-1 / 3}\left\{1 - \text { const } \left[\frac{1}{3} 2^{-1 / 3} c_{1}\left(1+\frac{3 \Pi_{b} r_{0}}{\sigma}\right)\right.\right. \\
& \times\left(\frac{(\sigma / \beta)^{1 / 3}}{r_{0}}\right)^{2} \Pi_{\mathrm{eff}}^{-4 / 3} \\
& \left.\left.+\frac{a}{3} 2^{(a+1) / 3} c_{2} B \xi_{b}^{1-a}\left(\frac{\Pi_{\mathrm{eff}}}{\sigma^{2} \beta}\right)^{(a-2) / 3}\right]\right\}
\end{aligned}
$$

Setting $\Pi_{b}=0$, we obtain

$$
\begin{aligned}
l_{W} \sim & \xi_{b}^{2 / 3} r_{0}^{1 / 3}\left\{1 - \text { const } \left[\frac{1}{3} 2^{-1 / 3} c_{1}\left(\xi_{b} / r_{0}\right)^{2 / 3}\right.\right. \\
& \left.\left.+\frac{a}{3} 2^{(a+1) / 3} c_{2} \tilde{B}(T)\left(\xi_{b} / r_{0}\right)^{(a-2) / 3}\right]\right\}
\end{aligned}
$$

where $\tilde{B}(T) \equiv B \xi_{b}^{1-a}$ is the dimensionless prefactor of $r_{0} \xi_{b}^{a-1} l^{-a}$ in the asymptotic form of $\beta u(l)$ [see Eq. (68)]. The two correction terms in Eq. (78) start being important as the size of $r_{0}$ approaches $\xi_{b}$ from above (if we consider $a=3$ or $4>2$ [see above Eq. (68)]) and provide the rate in which the mean thickness $l_{W}$ vanishes. In the case of $a=4$, the two correction terms are of the same order proportional to $r_{0}^{-1 / 3}$ and since $c_{1} \gg c_{2} \tilde{B}\left(T>T_{W}\right)$, where $T_{W}$ is the wetting temperature for the analogous planar system, we expect the first term to be dominant. When $a=3$ and $r_{0}>\xi_{b}$, $\left(\xi_{b} / r_{0}\right)^{2 / 3}<\left(\xi_{b} / r_{0}\right)^{(a-2) / 3}=\left(\xi_{b} / r_{0}\right)^{1 / 3}$. Hence, if $r_{0}$ is considerably larger than $\xi_{b}$, the second correction term proportional to $\tilde{B}$ becomes the dominant correction. In that limit, the asymptotic form of the interface potential (68) is valid, but at temperatures larger than $T_{W}$, the scaling form (37) and (38) tells us that the $\tilde{B}$ term is negligible. We thus expect the leading correction to the curvature controlled complete wetting behavior to be proportional to $r_{0}^{-1 / 3}$.

\section{CONCLUSIONS}

In conclusion, we have studied wetting of a disk in two dimensions. The direct motivation for the study is provided by the recent interest in the effects that protein inclusions in biomembranes could have a near first-order phase transition (or phase separation) in the membrane matrix. However, our results can be applied to any other two-dimensional wetting problem of a disklike substrate, while the phenomenological consideration, based on balancing the disjoining and the capillary pressure, can be perhaps extended to more complicated geometries.

An intuitively appealing description of this situation (Sec. II) is provided by the concept of pressure balance at the interface separating the wetting film from the matrix. For sufficiently thick films the likely source of the outward, disjoining pressure, known to play the dominant role in the two-dimensional wetting of a planar substrate, is the entropic repulsion between the interface and the substrate $\Pi_{d} \propto l^{-3}$ [see Eqs. (3) and (6)]. It is balanced by the curvature-induced Laplace pressure $\sigma / r$ 
(4), where $r=r_{0}+l$ is the radius of curvature of the interface. Correspondingly, in the limit of vanishing curvature of the substrate $r_{0} \rightarrow \infty$, the thickness of the film diverges as $r_{0}^{1 / 3}$ [see Eq. (8)].

The weak link in this argument is the extrapolation of the asymptotic law (6) obtained for the entropic repulsion of a planar substrate to the curved one. The problem is that the fluctuations of the interface are controlled by the first term in the Hamiltonian (16), which in the presence of curvature acquires a singular dependence on the fluctuations in the thickness. A dependence of the effective stiffness of the interface has been recently shown [16] to qualitatively change critical wetting behavior in three dimensions.

The transfer matrix analysis of this paper provides a direct way of generating a sequence of approximations for the quantities of interest in the small curvature limit. It turns out that the qualitative picture of Sec. II indeed correctly describes the leading scaling behavior at a complete wetting transition controlled by vanishing curvature. Moreover, scaling forms at the multicritical point, arising when a small curvature is added to the critical wetting point of a planar problem, are readily obtained from those of the planar problem by simply adding the Laplace pressure $\sim \sigma / r_{0}$ [Eqs. (42) and (59)] to the bulk thinning pressure value, resulting in the effective thinning pressure

$$
\Pi_{\mathrm{eff}}=\frac{\sigma}{r_{0}}+\Pi_{b}
$$

The curvature dependence of the stiffness term gives rise, however, to singular power-law corrections to the leading scaling behavior (78)

$$
l_{W} \sim \xi_{b}^{2 / 3} r_{0}^{1 / 3}\left(1-\text { const } \xi_{b}^{2 / 3} r_{0}^{-2 / 3}+\cdots\right)
$$

Such corrections can be crucial in obtaining a satisfactory fit of actual experimental data taken on a system of disks of finite radii.

\section{ACKNOWLEDGMENTS}

John H. Ipsen is deeply acknowledged for motivating our work on this problem as well as for useful discussions. One of us (T.G.) would also like to thank Martin J. Zuckerman for relevant discussions. The work of L.V.M. has been supported in part by the National Science Foundation under Grant No. PHY89-04035. This support came through the Santa Barbara ITP, whose hospitality is acknowledged.
[1] S. Dietrich, in Wetting Phenomena, in Phase Transition and Critical Phenomena, edited by D. Domb and J. Lebowitz (Academic, New York, 1988), Vol. 12.

[2] M. Schick, in Liquids at Interfaces, 1988, Les Houches Lectures, edited by J. Charvolin, J. F. Joanny, and J. Zinn-Justin (North Holland, Amsterdam, 1990).

[3] L. V. Mikheev and A. A. Chernov, Zh. Eksp. Teor. Fiz. 92, 1732 (1987) [Sov. Phys. JETP 65, 971 (1987)]; L. V. Mikheev, Phys. Rev. Lett. 69, 860 (1992).

[4] D. Beysens and D. Esteve, Phys. Rev. Lett. 54, 2123 (1985).

[5] O. G. Mouritsen and M. Bloom, Annu. Rev. Biophys. Biomol. Struct. 22, 145 (1993).

[6] O. G. Mouritsen and M. M. Sperotto, in Thermodynamics of Lipid-Protein, Interaction in Lipid Membranes: The Hydrophobic Matching Condition, in Thermodynamics of Membranes, Receptors and Channels, edited by M. B. Jackson (CRC, Boca Raton, FL, 1993).

[7] J. N. Israelachvili, Intermolecular and Surface Forces, 2nd ed. (Academic, New York, 1992).

[8] M. P. Gelfand and R. Lipowsky, Phys. Rev. B 36, 8725 (1987).

[9] P. J. Upton, J. O. Indekeu, and J. M. Yeomans, Phys. Rev. B 40, 666 (1989).

[10] G. A. Darbellay and J. M. Yeomans, J. Phys. A 23, 5655 (1990).

[11] M. E. Fisher, J. Stat. Phys. 34, 667 (1984).
[12] M. E. Fisher, J. Chem. Soc. Faraday Trans. 2 82, 1569 (1986).

[13] R. Lipowsky and M. E. Fisher, Phys. Rev. B 36, 2126 (1987).

[14] M. E. Fisher, in Fluctuations, Interactions and Related Transitions, Jerusalem Winter School for Theoretical Physics, edited by D. Nelson, T. Piran, and S. Weinberg (World Scientific, Singapore, 1989), Vol. 5.

[15] H. T. Dobbs, G. A. Darbellay, and J. M. Yeomans, Europhys. Lett. 18, 493 (1992).

[16] M. E. Fisher and A. J. Jin, Phys. Rev. Lett. 69, 792 (1992); A. J. Jin and M. E. Fisher, Phys. Rev. B 47, 7365 (1993); 48, 2642 (1993); M. E. Fisher, A. J. Jin, and A. O. Parry, Ber. Bunsenges. Phys. Chem. 98, 357 (1994).

[17] R. P. Feynman, Quantum Mechanics and Path Integrals (McGraw-Hill, New York, 1965).

[18] K. Huang, Statistical Mechanics, 2nd ed. (Wiley, New York, 1987).

[19] D. M. Kroll and R. Lipowsky, Phys. Rev. B 28, 5273 (1983).

[20] D. M. Kroll and G. Gompper, Phys. Rev. B 39, 443 (1989).

[21] L. D. Landau and E. M. Lifshitz, Quantum Mechanics, Non-Relativistic Theory, 2nd ed. (Pergamon, New York, 1975). 